\title{
Ab-initio Electrodynamic Modeling of On-Chip Back-End Structures
}

\author{
Wim Schoenmaker \\ IMEC \\ Kapeldreef 75, B-3001 Leuven, Belgium \\ schoen@imec.be \\ Peter Meuris \\ IMEC \\ Kapeldreef 75, B-3001 Leuven, Belgium \\ meurisp@imec.be \\ Wim Magnus \\ IMEC \\ Kapeldreef 75, B-3001 Leuven, Belgium \\ magnus@imec.be
}

\begin{abstract}
The geometrical structure of electrodynamics is reviewed following the analogy with gravity. It is found that the vector potentials that represent magnetic fields can be identified as connections. As a consequence, these potentials should be assigned to the links of discretization grids. A ghost field is introduced to guarantee numerical stability in the solution scheme of solving electromagnetic field problems for interconnects and on-chip passives.
\end{abstract}

\section{Introduction}

Present-day integrated circuits are characterized by the down-scaling of device dimensions, the densification of device stacking as well as increased operation frequency. Furthermore, the integration of on-chip passive elements in the backend process of the circuit design is a continuing activity. The high density device stacking combined with a higher clock frequency leads to unprecedented design difficulties because one needs to keep parasitic couplings below prescribed threshold levels (design rules). On the other hand, the integration of on-chip passives requires a detailed knowledge of the characteristic parameters such as the capacitance, inductance and resistance. These global parameters can be regarded as effective parameters representing objects that are obtained from integrating a local, i.e. space-time dependent quantity. As such, these parameters will be sensitive to the frequency under consideration.

In order to arrive at a full chip design, a hierarchical approach is advocated. The design-rule checking is done by partitioning the design into blocks and within each block one may zoom in to the critical paths and to study these sections in greater detail. The less critical parts can usually be handled by lumped-element descriptions or transmission line theory. The critical paths are sometimes referred to as 'tunnels' [1]. 
At the deepest level, the simulation of the back-end structures should be done by a field-solver approach. However, the circumstances at this level are rather unconventional from a field-solvers point of view.

We will summarize here the specific difficulties that a field solver should be able to deal with. Geometry (1) The structures that appear at this level are often essentially three dimensional. As a consequence, transmission-line theory is not applicable. Geometry (2) The patterns have a width and height of comparable size. Therefore, approaches that assume infinitely thin metallic layers are not justified from first principles. Materials (1) Metallic patterns will have finite resistivity and therefore the currents will flow with different densities in the metalic parts. These currents distributions are further modified by the skin effect and proximity effects if the frequency increases. Materials (2) Semiconducting materials are moderately conducting. This has a serious impact on the determination of effective parameters. The slow-wave mode is a consequence of putting a thin oxide layer between the metallic conductor and the semiconducting material.

Considering all the requirements listed above, we discuss in this paper a novel approach. Instead of building on existing approximation schemes derived from Maxwell's equations, we choosed to return to the Maxwell equations and to develop a solution scheme capable if dealing with the above problems. In addition it proves beneficial not only to return to the Maxwell equations, but also to carefully trace the physics that is hidden in the various symbols. In particular, the 'physical' identification of the vector potential is crucial for a successful numerical implementation. There exists an intimate connection between differential geometry and electrodynamics that we have to exploit in order to perform this task.

\section{The geometry of electrodynamics}

Electrodynamics was discovered as a phenomenological theory. Starting from early experiments one arrived after considerable effort at Gauss' law, BiotSavart's law and Faraday's law of induction. Only Maxwell's law was obtained by theoretical reasoning and later experimentally confirmed by Herz. Maxwell's great achievement was later equalized by Einstein who proposed in the general theory of relativity that gravity $\equiv$ curvature. Ever since Einstein's achievement of describing gravity in terms of non-Euclidean geometry, theoretical physics has witnessed a stunning development based on geometrical reasoning resulting into the gauge theories having a geometrical interpretation very analogous to Einstein's theory of gravity. Electrodynamics is the low-energy limit of these gauge theories. Besides the aesthetic beauty that results from these insights, there is also pragmatic benefit. Solving electrodynamic problems on the computer, guided by the geometrical meaning of the variables is a decisive factor for the success of the calculation. This was already realized by Wilson [2] who initiated computer calculations on the quantum aspects of gauge theories. In order to perform computer calculations of the classical fields, geometry plays an equally important role as will be shown here. 
The classical fields $\mathbf{E}$ and $\mathbf{B}$ as well as the sources $\rho$ and $\mathbf{J}$ are invariant under gauge transformations and therefore their underlying geometrical meaning is hidden. We may identify the proper geometric character for these variables, such as scalars (zero-forms), force fields (one-forms), fluxes (two-forms) or volume densities (three-forms) as could be done for any other fluid dynamic system. This can be done without reference to the geometric nature of electrodynamics in the sense that $\mathbf{E}$ and $\mathbf{B}$ represent the curvature in the geometrical interpretation of electrodynamics. Therefore, in this paper we will consider the scalar potential and vector potential fields that $d o$ depend on gauge transformations and as such will give access to the geometry of electrodynamics.

\subsection{Gravity as a gauge theory}

The history of the concept of gauge invariance begins with the discovery of the principle of general covariance in general relativity. According to this principle the physical laws should maintain their form for all coordinate systems. Weyl [3] made an attempt to unify electrodynamics with gravity. According to the general theory of relativity, the gravitational field corresponds to curvature of space-time, and therefore, if a vector is parallel transported along a closed loop, the angle between the starting vector and the final vector will differ from zero. Furthermore, this angle is a measure for the curvature in the space. Weyl extended the Riemann geometry in such a way that not only the angle changes but also the length of the vector. The relative change in length is described by an anti-symmetric tensor and this tensor is invariant under changing the "unit of length". This invariance is closely related to charge conservation. Weyl called this "Maszstab Invarianz". The theory turned out to be contradictory and was abandoned, but the term "Maszstab Invarianz" survived (Maszstab = measure = gauge). Within the realm of quantum mechanics the principle of gauge invariance obtained its final interpretation: gauge invariance should refer to the phase transformations that may be applied on the wave functions. In particular, the phase transformation may be applied with different angle for different points in space and time. At first sight it looks as if we have lost the geometrical connection and that the link is only historical. However, a closer look at gravity shows that the link is still present.

Starting from the idea that all coordinate systems are equivalent, we may consider a general coordinate transformation $x^{\mu} \rightarrow x^{\prime \mu}=x^{\prime \mu}\left(x^{\nu}\right)$ and the transformation rule for coordinate differentials $d x^{\prime \mu}=\frac{\partial x^{\prime \mu}}{\partial x^{\nu}} d x^{\nu}$. A tuple transforming under a change of coordinates as the coordinate differentials is a contravariant vector and a scalar transforms in an invariant way. The derivative of a scalar $V_{\mu}=\frac{\partial \phi}{\partial x^{\mu}}$ transforms as $V_{\mu}^{\prime}=\frac{\partial x^{\nu}}{\partial x^{\prime \mu}} V_{\nu}$. Any tuple transforming under a change of coordinates as the differentials of a scalar function is a covariant vector and in general, tensors transform according to a multiple set of pre-factors. The principle of general coordinate covariance can be implemented by claiming that all physical laws should be expressed as tensor equations. Since left and right hand side will transform with equal sets of pre-factors, their form invariance is guaranteed. 
So far, we have only been concerned with the change from one arbitrary coordinate system to another. One might argue that this will hide well-known results in a thick shell of notational complexity. In order to peal off this shell and to reveal the physical implications one must refer to the intrinsic properties of the geometric structure. Occasionally, the intrinsic structure is simple, e.g. flat space time, and the familiar relations are recovered. It was Einstein's discovery that space-time is not flat in the presence of matter and therefore the physical laws are more involved.

Riemann geometry is a generalization of Euclidean geometry in the sense that locally one can still find coordinate systems, $\xi^{\mu}=(i c t, \mathbf{x})$, such that the distance between two near-by point is given by Pythagoras' law, i.e. $d s^{2}=\delta_{\mu \nu} d \xi^{\mu} d \xi^{\nu}$. In an arbitrary coordinate system the distance is given by

$$
d s^{2}=g_{\mu \nu}(x) d x^{\mu} d x^{\nu}, g_{\mu \nu}(x)=\frac{\partial \xi^{\alpha}}{\partial x^{\mu}} \frac{\partial \xi^{\beta}}{\partial x^{\nu}} \delta_{\alpha \beta}
$$

In the local coordinate system, $\xi$, the equation of motion of a freely falling particle is $\frac{d^{2} \xi^{\mu}}{d s^{2}}=0$. In an arbitrary coordinate system, this equation becomes

$$
\frac{d^{2} x^{\alpha}}{d s^{2}}+\Gamma_{\mu \nu}^{\alpha} \frac{d x^{\mu}}{d s} \frac{d x^{\nu}}{d s}=0
$$

where $\Gamma_{\mu \nu}^{\alpha}$ is the affine connection also referred to as the Christoffel symbols.

$$
\Gamma_{\mu \nu}^{\alpha}=\frac{\partial x^{\alpha}}{\partial \xi^{\beta}} \frac{\partial^{2} \xi^{\beta}}{\partial x^{\mu} \partial x^{\nu}}
$$

The affine connection transforms under general coordinate transformations as

$$
\Gamma_{\mu \nu}^{\prime \alpha}=\frac{\partial x^{\prime \alpha}}{\partial x^{\rho}} \frac{\partial x^{\tau}}{\partial x^{\prime \mu}} \frac{\partial x^{\sigma}}{\partial x^{\prime \nu}} \Gamma_{\tau \sigma}^{\rho}+\frac{\partial x^{\prime \alpha}}{\partial x^{\rho}} \frac{\partial^{2} x^{\rho}}{\partial x^{\prime \mu} \partial x^{\prime \nu}}
$$

The second term destroys the covariance of the affine connection, i.e. the affine connection is not a tensor. The metric tensor $g_{\mu \nu}(x)$ contains information on the local curvature of the Riemann geometry. Now consider a vector $V^{\mu}(\tau)$ along a curve $x^{\mu}(\tau)$. In the local-Euclidean coordinate system $(\xi)$, the change of the vector along the curve is $d V^{\mu} / d \tau$. In another coordinate system $\left(x^{\prime}\right)$, we find from the transformation rules

$$
\frac{d V^{\prime \mu}}{d \tau}=\frac{\partial x^{\prime \mu}}{\partial x^{\nu}} \frac{d V^{\nu}}{d \tau}+\frac{\partial^{2} x^{\prime \mu}}{\partial x^{\nu} \partial x^{\lambda}} \frac{\partial x^{\lambda}}{\partial \tau} V^{\nu}(\tau)
$$

The second derivative in the second term is an inhomogeneous term in the transformation rule that prevents $d V^{\mu} / d \tau$ from being a vector and contains the key to curvature. This term, is directly related to the affine connection. The combination

$$
\frac{D V^{\mu}}{D \tau}=\frac{d V^{\mu}}{d \tau}+\Gamma_{\nu \lambda}^{\mu} \frac{d x^{\lambda}}{d \tau} V^{\nu}
$$


does transform as a vector and is called the covariant derivative along the curve. In the restricted region where we can use the Euclidean coordinates, $\xi$, we may apply Euclidean geometrical methods, and in particular we can shift a vector over an infinitesimal distance from one point to another and keep the initial and final vector parallel. The components of the vector do not alter by the shift operation: $\delta V^{\mu}=0$. Furthermore, in the local frame $x^{\mu}=\xi_{x(\tau)}^{\mu}$, the affine connection vanishes, i.e. $\Gamma_{\mu \nu}^{\alpha}=0$. Therefore, the conventional operation of parallelly shifting a vector in the locally-Euclidean coordinate system can be expressed by the equation $D V^{\mu} / D \tau=0$. Being a tensor equation, it holds for all coordinate systems. A vector, whose covariant derivative along a curve vanishes, is said to be parallel transported along the curve. The coordinates satisfy the following first-order differential equations.

$$
\frac{d V^{\mu}}{d \tau}=-\Gamma_{\nu \lambda}^{\mu} \frac{d x^{\lambda}}{d \tau} V^{\nu}
$$

The parallel transport of a vector $V^{\mu}$ over a small distance $d x^{\nu}$ changes the components of the vector by amounts $\delta V^{\mu}=-\Gamma_{\nu \lambda}^{\mu} V^{\nu} \delta x^{\lambda}$.

In general, if we want to perform the differentiation of a tensor field with respect to the coordinates, we must compare tensors in two nearby points. In fact, the comparison corresponds to subtraction, but a subtraction is only defined if the tensors are anchored to the same point. (In different points, we have different local coordinate systems.) Therefore we must first parallel transport the initial tensor to the nearby point before the subtraction can be performed. For example, the covariant differential of a vector field is

$$
D V^{\mu}=d V^{\mu}-\delta V^{\mu}=\left(\frac{\partial V^{\mu}}{\partial x^{\lambda}}+\Gamma_{\lambda \kappa}^{\mu} V^{\kappa}\right) d x^{\lambda}=D_{\lambda} V^{\mu} d x^{\lambda}
$$

So far, the general coordinate systems include both accelerations originating from non-uniform boosts of the coordinate systems as well as acceleration that may be caused by the gravitational field due to the presence of matter. In the first case, space-time is not really curved, in the second case it is curved. In order to find out whether gravitation is present one must extract information about the intrinsic properties of space-time. This can be done by the parallel transport of a vector field along a closed loop. If the initial and final vector differ, one can conclude that gravity is present. The difference that a closed loop transport generates is given by

$$
\Delta V^{\mu}=R_{\rho \lambda \sigma}^{\mu} V^{\rho} \delta x^{\lambda} \delta x^{\sigma}
$$

where $R_{\rho \lambda \sigma}^{\mu}=\Gamma_{\sigma \mu, \nu}^{\rho}-\Gamma_{\sigma \nu, \mu}^{\rho}+\Gamma_{\lambda \nu}^{\rho} \Gamma_{\sigma \mu}^{\lambda}-\Gamma_{\lambda \mu}^{\rho} \Gamma_{\sigma \nu}^{\lambda}$ is the curvature tensor or Riemann tensor. This tensor describes the intrinsic curvature in a point.

We are now prepared to consider the geometrical basis of electrodynamics and other gauge theories but we will first summarize a few important facts: (1) in each space-time point a local frame may be erected, (2) the affine connection is a path-dependent quantity, (3) the affine connection does not transform as 
a tensor, (4) the field strength (curvature) may be obtained by performing a parallel transport along a closed loop.

\subsection{The geometrical interpretation of electrodynamics}

Just as with the local Euclidean coordinate systems, we will consider the possibility to set up in each space-time point a local frame for fixing the phase of the complex wave function $\psi(\mathbf{x}, t)$. Since the choice of such a local frame (gauge) is not unique we may rotate this frame without altering the physical content of the wave function. We can guarantee the latter by demanding appropriate transformation properties (c.f. the tensors in above section) of the variables. Changing the local frame for the phase of a wave function amounts to

$$
\psi^{\prime}(\mathbf{r}, t)=\exp \left(\frac{i e}{\hbar} \chi(\mathbf{r}, t)\right) \psi(\mathbf{r}, t), \psi^{\prime *}(\mathbf{r}, t)=\exp \left(-\frac{i e}{\hbar} \chi(\mathbf{r}, t)\right) \psi^{*}(\mathbf{r}, t)
$$

These transformation rules are similar to the contravariant and covariant transformation rules for vectors in the foregoing section. We can construct a 'scalar' by taking $\psi^{*} \psi$. The derivative of the wave function transforms as

$$
\frac{\partial \psi^{\prime}}{\partial x^{\mu}}=\exp \left(\frac{i e}{\hbar} \chi\right) \frac{\partial \psi}{\partial x^{\mu}}+\frac{i e}{\hbar} \frac{\partial \chi}{\partial x^{\mu}} \exp \left(\frac{i e}{\hbar} \chi\right) \psi
$$

The second term prevents the derivative of $\psi$ from transforming as a 'vector' under the change of gauge. However, now geometry will be of help to provide a solution for constructing gauge covariant variables from derivatives. We must postulate an 'affine connection', such that a covariant derivative can be made. For that purpose a connection $A_{\mu}$ is defined that transforms as $A_{\mu}=A_{\mu}+\frac{\partial \chi}{\partial x^{\mu}}$. The covariant derivative is

$$
D_{\mu}=\frac{\partial}{\partial x^{\mu}}+\frac{i e}{\hbar} A_{\mu}
$$

Just as with the gravitational affine connection, the field $A_{\mu}$ can be used to construct 'parallel' transport. Therefore, the field $A_{\mu}$ must be assigned to the paths along which the transport takes place. The curvature of the connection can also be revealed by integrating the affine connection around a closed loop. The result is $F_{\mu \nu} \delta x^{\mu} \delta x^{\nu}=\oint d x^{\mu} A_{\mu}$ and $F_{\mu \nu}=\frac{\partial A_{\mu}}{\partial x^{\nu}}-\frac{\partial A_{\nu}}{\partial x^{\mu}}$ is the electromagnetic field tensor.

\section{Implications for numerical simulations}

In order to perform numerical computations starting from the fields $A_{\mu}$ it is necessary to introduce a discretization grid. The simulation of a finite space and/or space-time domain requires that each grid point is separated a finite distance from its neighboring points. Then the differential operators that appear in the continuous field equations must be translated to the discretization grid by proper reference to the geometrical meaning of the variables. The connections $A_{\mu}$ should be assigned to the links in the grid. 
The geometrical interpretation suggests that the link assignment is the only correct scheme for solving vector-potential field problems on the computer.

The numerical consequences of the above assignment will be considered in the following example, in which we will solve the steady-state equations $\nabla \times \mathbf{B}=$ $\mu_{0} \mathbf{J}, \mathbf{B}=\nabla \times \mathbf{A}, \mathbf{J}=\sigma \mathbf{E}, \mathbf{E}=-\nabla V$. The usual approach consists of substituting the second equation into the first one, adopting the Coulomb gauge $\nabla \cdot \mathbf{A}=0$ and obtaining $-\nabla^{2} \mathbf{A}=\mu_{0} \mathbf{J}$. Analytic solution schemes address this equation as a three-fold Poisson equation. This approach is usually sustained in numerical solution schemes, assigning the three components $A_{x}, A_{y}, A_{z}$ to the nodes of the discrete lattice. This approach is in conflict with the geometrical interpretation of electrodynamics, since three scalar fields and the three components of a vector field are geometrically different objects.

We will now discretize the set of equations on a regular Cartesian grid having $N$ nodes in each direction following our new approach. The total number of nodes in $D$ dimensions is $M_{\text {nodes }}=N^{D}$ and the number of links in the lattice is $M_{\text {links }}=D N^{D}\left(1-\frac{1}{N}\right)$.

As far as the description of the electromagnetic field is concerned, the counting of unknowns for the full lattice results into $M_{\text {links }}$ variables $\left(A_{i j}\right)$ for the links, and $M_{\text {nodes }}$ variables $\left(V_{i}\right)$ for the nodes. Since each link (node) gives rise to one equation, the naive counting is consistent. However, we have not yet implemented the gauge condition. The conventional Coulomb gauge $\nabla \cdot \mathbf{A}=0$, constraints the link degrees of freedom and therefore not all link fields are independent. There are $3 N^{3}\left(1-\frac{1}{N}\right)$ link variables and $3 N^{3}\left(1-\frac{1}{N}\right)+N^{3}$ equations, including the constraints. As a consequence, at first sight it seems that we are confronted with an overdetermined system of equations, since each node provides an extra equation for $\mathbf{A}$. However, the translation of the Maxwell-Ampère equation on the lattice leads to a singular matrix, i.e. not all rows are independent. The rank of the corresponding matrix is $3 N^{3}\left(1-\frac{1}{N}\right)$, whereas there are $3 N^{3}\left(1-\frac{1}{N}\right)+N^{3}$ rows and $3 N^{3}\left(1-\frac{1}{N}\right)$ columns. Such a situation is highly inconvenient for solving non-linear systems of equations, where the non-linearity stems from the source terms being explicitly dependent on the fields. Moreover, the application of the Newton-Raphson method requires that the matrices in the Newton equation be non-singular and square. In fact, the non-singular and square form of the Newton matrix can be recovered by introducing the more general gauge $\nabla \cdot \mathbf{A}+\nabla^{2} \chi=0$, where an additional scalar field $\chi$, i.e. one unknown per node, is included. In this way the number of unknowns and the number of equations match again. In the continuum limit $(N \rightarrow \infty)$, the field $\chi$ and one component of $\mathbf{A}$ can be eliminated.

Though being irrelevant for theoretical understanding, the auxiliary field $\chi$ is essential for obtaining numerical stability on a discrete, finite lattice.

In other words, our specific gauge solely serves as a tool to obtain a discretization scheme that generates a regular Newton-Raphson matrix. 
To summarize, instead of solving the problem

$$
\nabla \times \nabla \times \mathbf{A}=\mu_{0} \mathbf{J}(\mathbf{A}), \nabla \cdot \mathbf{A}=0
$$

we solve the equivalent system of equations

$$
\nabla \times \nabla \times \mathbf{A}-\gamma \nabla \chi=\mu_{0} \mathbf{J}(\mathbf{A}), \nabla \cdot \mathbf{A}+\nabla^{2} \chi=0
$$

The equivalence of both sets of equations (13) and (14) was be demonstrated by [4]. Physical equivalence is guaranteed provided that $\nabla \chi$ does not lead to an additional current source. Therefore, it is required that $\nabla \chi=0$. In fact, acting with the divergence operator on the first equation of (14) gives Laplace's equation for $\chi$. The solution of the Laplace equation is identically zero if the solution vanishes at the boundary. We achieved to implement the gauge condition resulting into a unique solution and simultaneously to arrive at a system containing the same number of equations and variables. Hence a regular square NewtonRaphson matrix is obtained at each stage of the numerical solution scheme that solves the full set of non-linear equations.

\subsection{Discretized operators}

Integrated over a test volume $\Delta V_{i}$ surrounding a node $i$, the divergence operator, acting on vector potential $A$, can be discretized as a combination of 6 neighboring links

$$
\int_{\Delta V_{i}} \nabla \cdot \mathbf{A} d \tau=\int_{\partial\left(\Delta V_{i}\right)} \mathbf{A} \cdot \mathbf{d} \mathbf{S} \sim \sum_{k}^{6} S_{i k} A_{i k}
$$

The symbol $\sim$ represents the conversion to the grid formulation and $\partial\left(\Delta V_{i}\right)$ denotes the boundary of $\Delta V_{i}$.

The curl-curl operator can be discretized for a link $i j$ using a combination of 12 neighboring links and the link $i j$ itself. As indicated in Fig. 1, the field $\mathbf{B}_{i}$ in the center of the 'wing' $i$, can be constructed by taking the circulation of the vector potential $A$ around the wing $i(i=1,4)$

$$
\mathbf{B}_{i} S_{i}=\sum_{j=1}^{3} \mathbf{A}_{i j} h_{i j}+\mathbf{A}_{0} h_{0}
$$

where $h_{\alpha}$ is the length of the corresponding link $\alpha$. Integration over a surface $S_{i j}$ perpendicular to the link $i j$ yields a linear combination of different $\mathbf{A}_{i j}$ 's, the coefficients of which are denoted by $\Lambda_{i j}$.

$$
\begin{aligned}
\int_{\Delta S_{i j}} \nabla \times \nabla \times \mathbf{A} \cdot \mathbf{d} \mathbf{S} & =\int_{\partial\left(\Delta S_{i j}\right)} \nabla \times \mathbf{A} \cdot \mathbf{d} \mathbf{r} \\
= & \int_{\partial\left(\Delta S_{i j}\right)} \mathbf{B} \cdot \mathbf{d} \mathbf{r}
\end{aligned}
$$

For the nodal variables we apply the usual discretization methods. 


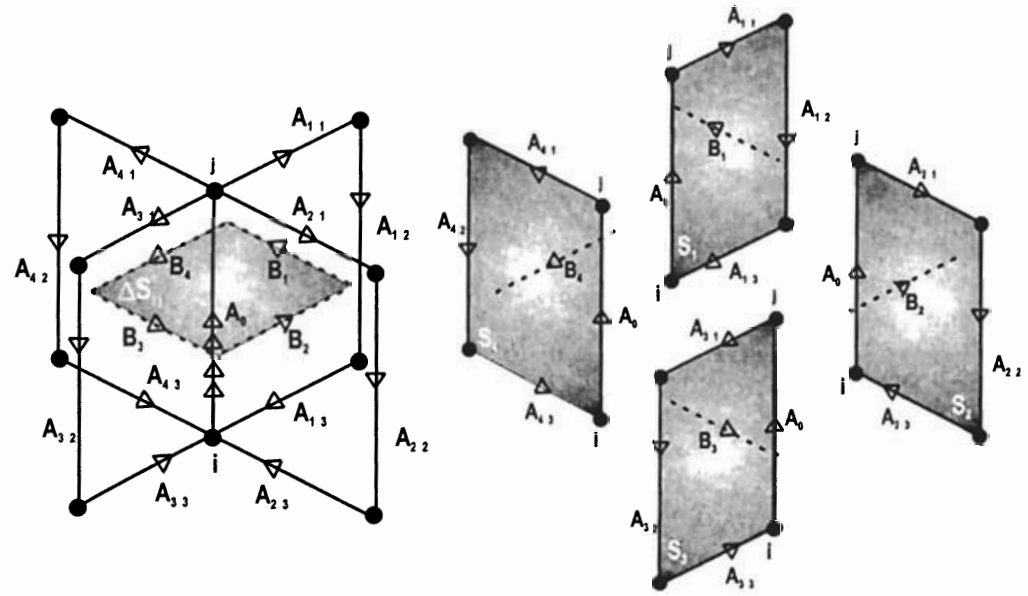

Figure 1: The assembly of the $\nabla \times \nabla \times$-operator using 12 contributions of neighboring links.

\section{Application to the Spiral Inductor}

We present an example demonstrating that the proposed potential formulation in terms of the Poisson field $V$, the vector field $\mathbf{A}$ and the ghost field $\chi$, is a viable method to solve the Maxwell field problem. All subtleties related to that formulation, i.e. the positioning of the vector potential on links, and the introduction of the ghost field $\chi$, are already encountered in constructing the solutions of the static equations.

A spiral inductor, as shown in Fig. 2 (left) was simulated. This structure also addresses the three dimensional aspects of the solver. The cross-section of the different lines is $1 \mu \mathrm{m} \times 1 \mu \mathrm{m}$. The overall size of the structure is $8 \mu \mathrm{m} \times 8$ $\mu \mathrm{m}$ and the simulation domain is $23 \times 20 \times 9 \mu \mathrm{m}^{3}$. The resistance is evaluated as $R=V / I$ and is $0.54 \Omega$. In Fig. 2 (right), the intensity of the magnetic field is shown at height $4.5 \mu \mathrm{m}$. From the results in Table II we obtain that the inductance of the spiral inductor is $4.23 \times 10^{-11}$ Henry.

\begin{tabular}{|c|c||c|c|}
\hline \multicolumn{2}{|c||}{ Electric energy $(J)$} & \multicolumn{3}{c|}{ Magnetic energy $(J)$} \\
\hline$\frac{1}{2} \epsilon_{0} \int d \tau E^{2}$ & $2.22 \mathrm{E}-18$ & $\frac{1}{2 \mu_{0}} \int d \tau B^{2}$ & $3.80 \mathrm{E}-13$ \\
$\frac{1}{2} \int d \tau \rho \phi$ & $2.35 \mathrm{E}-18$ & $\frac{1}{2} \int d \tau \mathbf{J} \cdot \mathbf{A}$ & $3.90 \mathrm{E}-13$ \\
\hline
\end{tabular}

Table II: Some characteristic results for the spiral inductor.

\section{Conclusions}

We reviewed the geometrical aspects of electromagnetic field variables. Vector potentials are 1 -forms. This has severe consequences for their discretization. The vector potentials need to be assigned to the links of the grid. Inclusion 

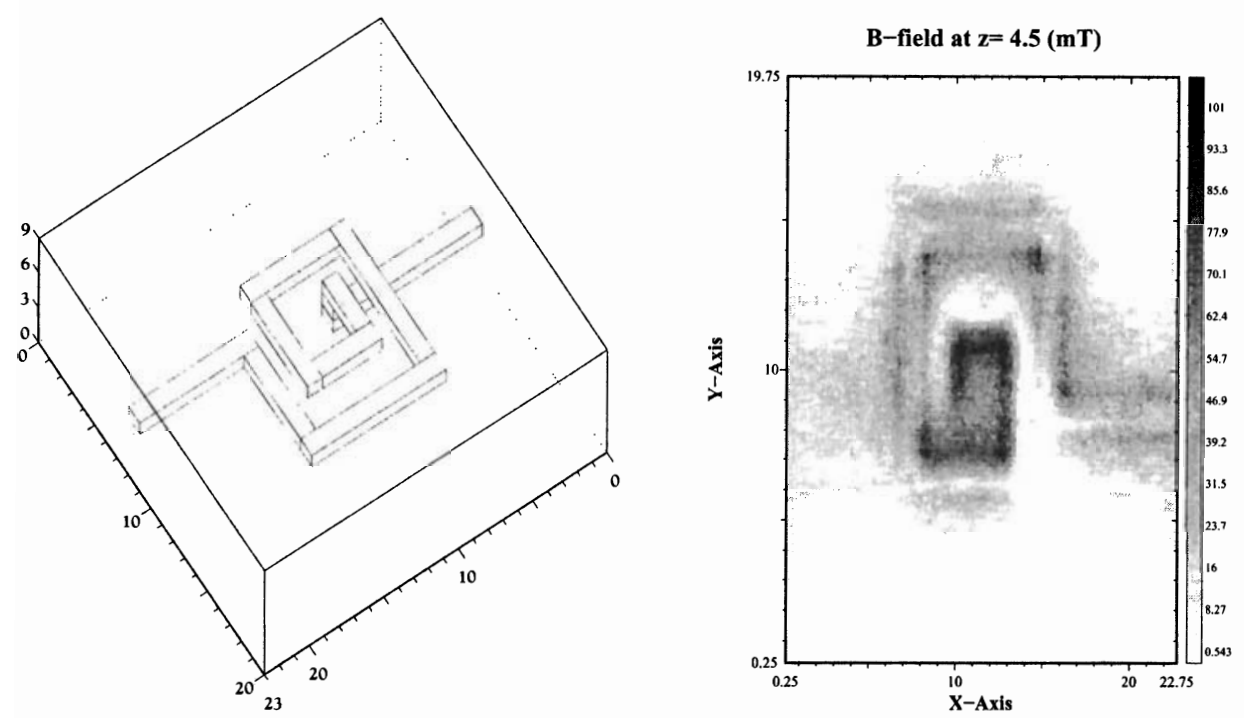

Figure 2: Layout of the spiral inductor structure (left) and the magnetic field strength in the plane of the spiral inductor (right).

of the gauge condition gives rise to non-symmetric Newton-Raphson matrices, when solving non-linear electromagnetic problems. This can be repaired by the inclusion of a 'ghost' field that has no physical effects but results into nonsingular and square Newton-Raphson matrices.

Acknowledgement Part of this work is funded by the Flemish Institute for Science and Technology (IWT), Flanders (Belgium).

\section{References}

[1] Walker M.G. Modeling the wiring of deep submicron IC. IEEE Spectrum 2000; March edition.

[2] Wilson K. Confinement of Quarks. Phys. Rev. 1974; D10: 2445-2459

[3] Weyl H. Gravitation und Elektrizität, Sitzungberichte der KöniglichPreussischen Akademie der Wissenschaften. 1918; 26: 465-480

[4] Meuris P., Schoenmaker W., Magnus W. Strategy for Electromagnetic Interconnect Modeling. IEEE Trans. on CAD 2000; Vol. 20 No. 6 